High Energy Phenomena in Relativistic Outflows III (HEPRO III)

International Journal of Modern Physics: Conference Series

Vol. 8 (2012) 241-252

(C) World Scientific Publishing Company

DOI: $10.1142 / \mathrm{S} 2010194512004667$

\title{
JET STABILITY, DYNAMICS AND ENERGY TRANSPORT
}

\author{
MANEL PERUCHO \\ Departament d'Astronomia i Astrofísica, Universitat de València, \\ C/ Dr. Moliner 50 Burjassot, Valencian Country, 46100, Spain \\ manel.perucho@uv.es
}

\begin{abstract}
Relativistic jets carry energy and particles from compact to very large scales compared with their initial radius. This is possible due to their remarkable collimation despite their intrinsic unstable nature. In this contribution, I review the state-of-the-art of our knowledge on instabilities growing in those jets and several stabilising mechanisms that may give an answer to the question of the stability of jets. In particular, during the last years we have learned that the limit imposed by the speed of light sets a maximum amplitude to the instabilities, contrary to the case of classical jets. On top of this stabilising mechanism, the fast growth of unstable modes with small wavelengths prevents the total disruption and entrainment of jets. I also review several non-linear processes that can have an effect on the collimation of extragalactic and microquasar jets. Within those, I remark possible causes for the decollimation and deceleration of FRI jets, as opposed to the collimated FRII's. Finally, I give a summary of the main reasons why jets can propagate through such long distances.
\end{abstract}

Keywords: relativistic jets; magnetohydrodynamics; instabilities

\section{Introduction}

Relativistic jets in AGN and microquasars carry energy from very small to very large scales. These jets form in the surroundings of compact objects, such as neutron stars or stellar black-holes in the case of microquasar jets, and supermassive blackholes $(\mathrm{SMBH})$ in the case of AGN jets. The forming scales are of the order of a few times to about 30 times the radius of the central object, depending on the forming mechanism. ${ }^{1,2}$ From these regions, jets propagate up to nine powers of ten in distance. This implies formidable stability and collimation taking into account that different instabilities can affect the evolution of jets independently of their nature. Moreover, their propagation is possibly changing the properties and evolution of the host galaxies and their environments through heating by shocks and/or mixing, and removing of gas by shocks and transfer of momentum. Thus, jet stability has been a matter of study during the last four decades. ${ }^{3,4,5,6}$

Depending on the nature of jets, i.e., whether they are magnetically or particle dominated, different types of instabilities can arise. In the case of magnetically dominated jets, both current-driven (CDI) and Kelvin-Helmholtz (KHI) instabilities 
can grow, whereas in the case of particle dominated jets, only the latter may be present. ${ }^{7}$

Different stabilising mechanisms for both kinds of instabilities have been proposed, and some of them could explain the remarkable collimation and long distances covered by extragalactic jets, as opposed to the view that the reason for the stability of jets remains unknown. ${ }^{8}$

In this text I review the state-of-the-art of relativistic jet stability. ${ }^{\text {a }}$ Section 2 is focused on the CDI. Section 3 is devoted to the KHI. In Section 4 I overview several non-linear processes that can also dramatically affect the evolution of jets and their collimation. Finally, in Section 5 I discuss the possible reasons why relativistic jets manage to carry large amounts of energy from the central regions of galaxies or binary stars to very large distances.

\section{The sub-parsec scales: Current-Driven instability}

Relativistic jets are thought to be formed by magnetohydrodynamical processes in the surroundings of compact objects. ${ }^{1,2}$ Thus, it is reasonable to expect that jets are magnetically dominated close to these regions. ${ }^{7}$ In this regime, CDI is triggered by differences in the magnetic forces when a toroidal field is present. In the case that a pinch is produced, the larger force produced by the compressed lines allows the pinch to grow. In the case that a kink is produced, the magnetic lines get closer in the inner part of the kink, increasing the magnetic force and enlarging the curvature of the jet. Both modes are stabilised by the presence of a poloidal field component, as the magnetic tension of these lines acts against the compression or distortion of the jet.

CDI in magnetized flows has been studied from an analytical perspective in the non-relativistic regime by a number of authors. ${ }^{7}$ Results show that this instability becomes dominant at large values of helicity (ratio between the toroidal and poloidal field components) and it is stabilised by a strong poloidal magnetic field. Under the physical conditions typical of jets close to the forming regions, CDI is expected to be dominant, though with small growth rates. ${ }^{7}$ In the relativistic regime, numerical simulations of static columns have shown that growth rates decrease with decreasing Alfvén speed and, regarding the non-linear regime, an increasing helicity of the magnetic field with increasing radius in the jet, stabilises the flow with respect to kink CDI. ${ }^{9}$ The introduction of a sub-Alfvénic velocity shear generates important differences in the growth of this instability: In the case that the shear is inside the characteristic radius of the static column, the plasma flows through a temporally growing kink, whereas if the shear is outside that radius, the kink is advected with the flow and grows in distance. ${ }^{10}$

It has been suggested that the conversion from Poynting to kinetic flux should occur in the first hundreds of gravitational radii, on the basis of observations and

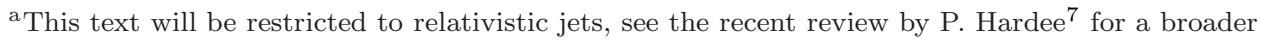
view including studies for classical jets 
modelling of the jet in M87.7,11,12 Magnetic acceleration has been proposed $^{13}$ as an efficient mechanism. The growth of CDI to non-linear amplitudes can result in mass-load and acceleration of the entrained particles, so this process could also be related to a change from magnetically to particle dominated jets. ${ }^{7}$ However, there is no direct evidence for any of both. The role of CDI could be significantly reduced by jet expansion and rotation, which can have a stabilising effect. Further simulations with realistic conditions are needed to solve this question. Nevertheless, extragalactic jets embed stars and are necessarily entrained by gas clouds and stars rotating around the AGN, so the mass-load and conversion of jet main energy channel from Poynting flux to particles is difficult to avoid. It has been shown that sub-Alfvénic flows are KHI stable, ${ }^{14}$ but once the jets are super-Alfvénic and particle dominated, KHI may take over CDI and start to play an important role in the long-term stability of jets.

\section{The parsec scales: Kelvin-Helmholtz instability}

The KHI develops at the boundary between two fluids with relative velocity. It couples to any perturbation with a certain periodicity at which the system presents unstable modes. The sound-wave generated by the perturbation propagates through the body of the jet and grows in amplitude due to over-reflection at the boundaries. ${ }^{15}$ In the non-linear regime, the jet can be disrupted and decelerated by the entrainment of external matter.

Assuming cylindrical symmetry in jets, solutions to the linearised equations of relativistic hydrodynamics have the form $\exp (i(\mathbf{k} \mathbf{r}+n \phi-\omega t)),{ }^{16} \mathbf{k}$ being the wavenumber, $n$ the azimuthal wave-number (an integer giving the number of oscillations around the jet's circular cross-section), and $\omega$ the frequency. These solutions may have complex values of wave-number and/or frequency, the imaginary part giving the exponential growth of the amplitude. Unstable modes are separated into surface modes and body modes. Their distinctive property is the number of zeros that the the radial component of the wave-number $\left(k_{r}\right)$ of the unstable wave (e.g., the pressure wave) has between the jet axis and its boundary. The surface mode shows no zeros between the axis and the boundary, whereas the body modes show as many zeros as their order indicates (e.g., one zero in the case of the first body mode). Figure 1 shows some extracted modes from the solution to the linear problem of a sheared jet. The upper lines represent the real part of the solution (frequency) versus the wave-number, whereas the lower lines represent the imaginary part (growth rate) versus wave-number.

The linear regime The growth of KHI has been shown to depend on the jet velocity (unstable modes grow faster for slower jets), temperature (faster growth for hotter jets) and density (faster growth for more dilute jets). ${ }^{3,4,5,17}$ There is a general correlation between this dependence and the long-term stability properties of jets, which is more remarkable in colder, faster and denser ones. ${ }^{18}$ 


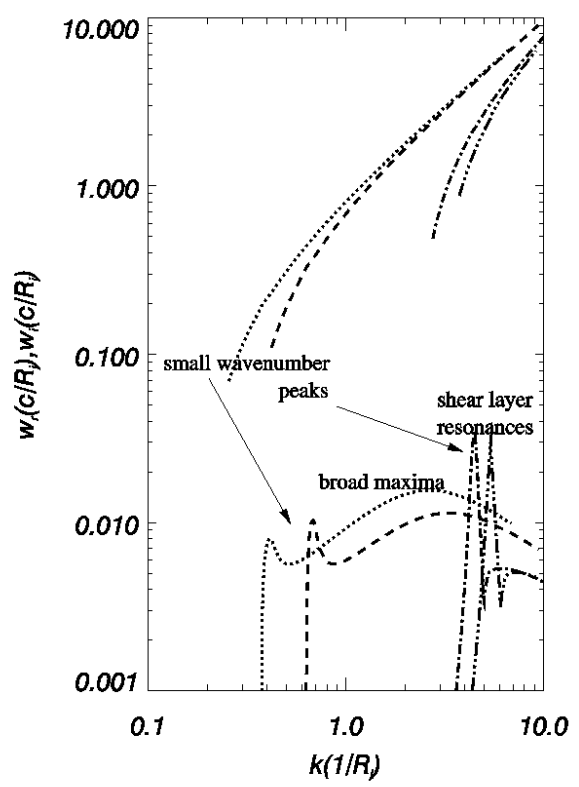

Fig. 1. Selected modes of a solution for a jet with a shear-layer. The plot shows frequency (real part of the solution, upper lines) and growth rates (imaginary part of the solution, lower lines) versus wave-number in units of speed of light and jet radius. Lines with the same format correspond to the real and imaginary part of the selected mode. Resonant modes show larger growth rates. From ref. 33 .

The analytical, and numerical in some cases, knowledge acquired about the effect that the growth of KHI modes could have on jets ${ }^{16,19}$ has been widely used in analysing the structures observed in different extragalactic jets and has allowed to derive estimates of the physical parameters that govern them (e.g., 3C 345, 3C 120, 3C 273, M87, S5 0836+710). ${ }^{12,19-26}$ In particular, it was shown that expansion and acceleration can provide the jet in $3 \mathrm{C} 120$ with long-term stability ${ }^{21}$ or that the jet in M87 could be decelerating and heating, leading to destabilization in the kpc scales. ${ }^{12}$ Numerical plus analytical work has also led to the conclusion that the observed structures in the jet in 3C 273 could be coupling to KHI modes, which may show up at different observing frequencies, depending on the section of the jet where they develop. ${ }^{22}$ This result also has the implicit conclusion that different observing frequencies may be showing different regions of a transversally structured jet. $^{24,26}$ In this respect, several works have also shown that shear-layers are easily generated in jets by different mechanisms, including the growth of KHI modes. ${ }^{18,27}$

In the classical regime, there is no limit to the growth of KHI, other than disruption of the jet or the saturation of short wavelengths (the latter will be discussed in the section about the non-linear phase), both involving an increase of the width of the velocity shear with a decrease of the radial gradient of velocity. ${ }^{28}$ For the case of relativistic jets, there is an analytical prediction of the saturation of the linear 


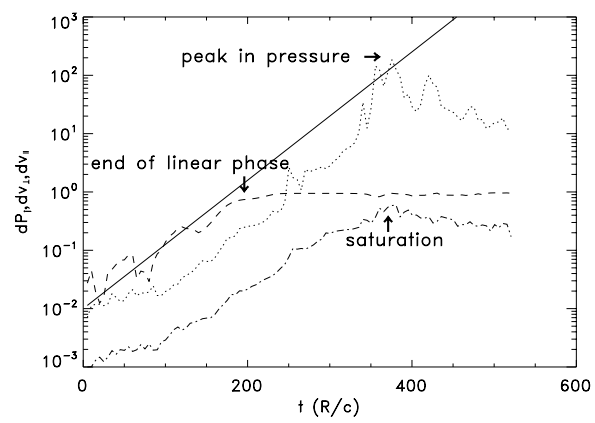

Fig. 2. Plot of the linear growth of KHI modes in a relativistic flow versus time. The dotted line represents the amplitude of the pressure perturbation and the dashed and dash-dotted lines represent the amplitude of the perturbation of the axial and radial components of velocity, respectively, in the reference frame of the jet. The units are speed of light, jet radius and ambient medium density. From ref. 31.

growth when the amplitude of the velocity perturbation reached the speed of light in the reference frame of the jet. ${ }^{29,30}$ Figure 2 shows the saturation of the perturbation in velocity close to the speed of light. Numerical simulations have confirmed this result and shown that the limiting amplitude is smaller for faster jets, thus making them more stable. ${ }^{31,32}$ Overall, this shows that relativistic jets are intrinsically more stable than classical ones with respect to the growth of any kind of instability.

On top of this, semi-analytic plus numerical studies of sheared jets have also shown that the growth of small-wavelength, fast-growing modes, as those shown on the right of Fig. 1, ${ }^{18,33}$ can lead to long-term collimation of fast jets with moderately thick shear-layers $\left(\simeq 1 R_{j}\right.$, with $R_{j}$ the jet radius $)$.

Different analytical and numerical works have certified that the presence of winds surrounding the jet ${ }^{34}$ or thick shear-layers ${ }^{24,35}$ reduce the growth rates of the KHI modes. It has also been shown analytically that in the presence of a poloidal magnetic field, sub-Alfvénic and even super-Alfvénic jets can be stabilised by the presence of a magnetised surrounding sheath. ${ }^{36}$

The non-linear regime starts typically after saturation. ${ }^{31,32}$ If the dominating mode is a low-order one with a relatively long wavelength, the jet shows strong deformations and can be disrupted by the generation of shocks at the boundary with the ambient medium. ${ }^{18,32}$ In this context, the jet develops a wide mixing layer and undergoes significant deceleration to mildly relativistic speeds. Otherwise, if small-wavelength modes like resonant modes (see Fig. 1) dominate the growth of the instability, the jet develops a hot shear-layer and keeps collimation. ${ }^{18,33}$ Figure 3 shows the Schlieren plots for the last snapshot of two simulations of the temporal growth of KHI in jets with different Lorentz factors $(\gamma=5$ for the left image and $\gamma=20$ for the right one). Otherwise, both simulations are equal. The left panel, 

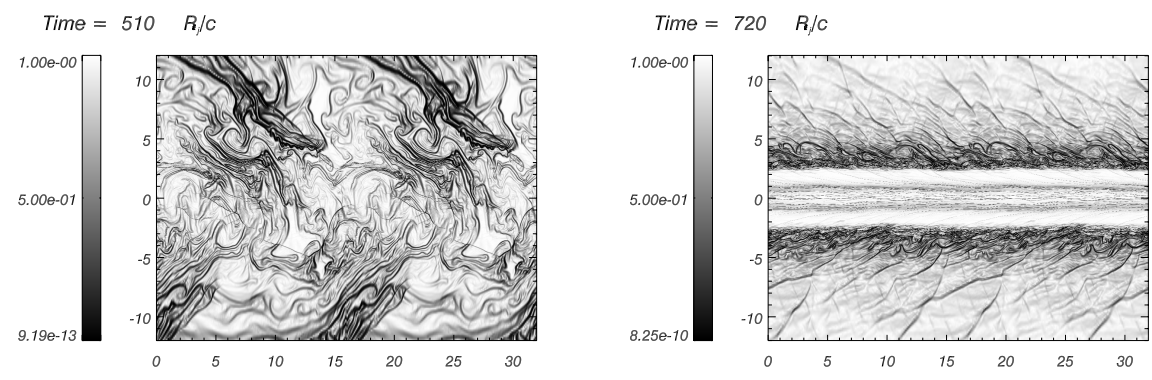

Fig. 3. Schlieren (enhanced density gradients) plots of two jets, one developing long wavelength modes (left) and another developing resonant (short-wavelength) modes. Axis are jet radii. From ref. 33 .

corresponding to a slower jet developing long-wavelength modes, shows turbulent mixing in a wide region, whereas the right panel, corresponding to the faster jet, which develops resonant modes, shows a significant degree of collimation. This result has been recently confirmed by $3 \mathrm{D}$ simulations and its implications extensively discussed $^{37}$ (see Figure 4). A similar, although not so efficient, mechanism for longterm jet stabilisation was also found for classical jets when the shortest modes dominate the growth of the instability up to the non-linear regime, ${ }^{38}$ which is also true for relativistic jets. ${ }^{32}$

The distribution of a number of simulated jets in a relativistic Mach-number versus Lorentz factor plane ${ }^{18,31,32}$ shows that there is a trend of larger stability in the non-linear regime in the case of faster and colder jets, which fall in the upperright corner of the plane.

\section{Non-linear effects}

In the previous section, the growth of instabilities has been discussed as starting in the linear regime, i.e., from small perturbations. However, jets are destabilised by perturbations with large (non-linear) amplitudes and may undergo reconfinement shocks or meet irregularities in the ambient media. These processes are non-linear and cannot be accounted for by linear theory of jet stability. They are of interest because generally imply strong shocks and thus represent candidate locations for very-high energy emission.

Extragalactic jets Most probably jets are not in pressure equilibrium with the ambient medium because the pressure in the surrounding cocoon changes with time, as it expands. Over-pressured jets expand and recollimate after becoming underpressured with respect to the environment, ${ }^{39,40}$ or meet high density regions in an inhomogeneous environment (e.g., supernova remnants (SNR) or massive stars 


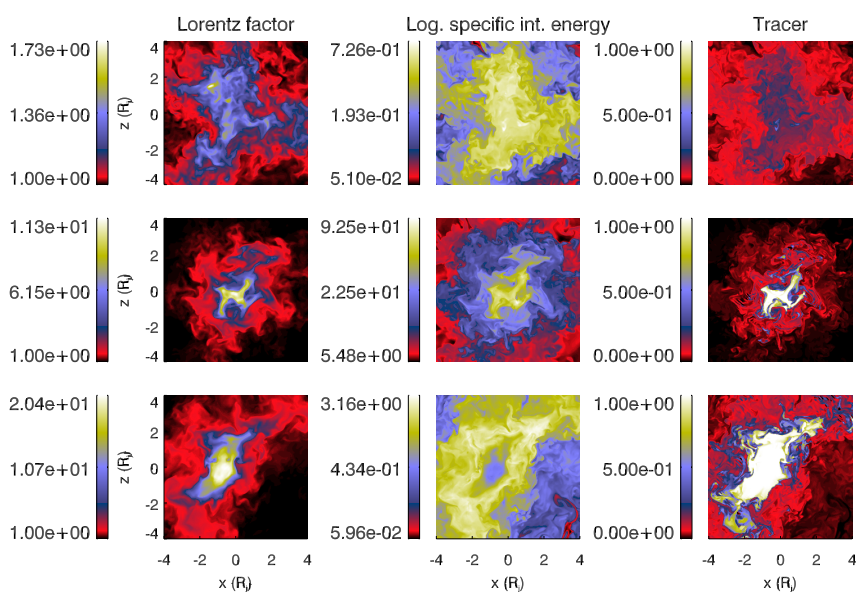

Fig. 4. Transversal cuts from 3D simulations of a cold jet with Lorentz factor 5 (top row) and 20 (bottom row) and an hot jet with Lorentz factor 10 (middle row). The cuts show Lorentz factor, logarithm of the specific internal energy (units of $c^{2}$ ) and jet mass fraction wrt original, from left to right. The faster jet shows $(\gamma=20$, bottom row) less mixing, preserves a larger Lorentz factor and develops a hot shear-layer, as predicted by the $2 \mathrm{D}$ simulations. The slow jet $(\gamma=5$, top row) undergoes strong mixing and deceleration, whereas the hot jet ( $\gamma=10$, top row) shares properties with both previous cases. From ref. 37.

with powerful winds). Depending on the initial pressure difference, these shocks can trigger small amplitude pinching or helical motion that may couple to $\mathrm{KHI},{ }^{41}$ or they can generate a large amplitude pinch and destroy the jet ${ }^{42}$ (see Figure 5). Only in the case that jets are close to pressure equilibrium with respect to the ambient medium, the generation and influence of reconfinement shocks can be neglected.

When crossing the galaxy, the jet flow may encounter stars and clouds of gas that can be embedded in the jet or entrain it due to their proper motions. ${ }^{43,44}$ In the case of stars, the stellar wind has been claimed to efficiently mix with the jet flow and decrease the mean jet velocity, ${ }^{45,46,47}$ mainly in the case of massive stellar winds and very light jets. Also, irregularities in the intergalactic medium have been claimed to play a role in the hybrid morphology of a number of sources that show both FRI and FRII structure in their two jets. ${ }^{48,49}$

Large amplitude initial perturbations, such as those triggered by changes in the injection angle of the flow could generate non-linear distortions of jets and lead to their disruption. ${ }^{50}$ The injection of overdense plasma in jets has been invoked to explain the observation of components travelling through parsec-scale jets and the generation of trailing components. ${ }^{41,51,52,53}$ These have been shown to generate significant pinching that could couple to KHI modes. ${ }^{41}$ In the kiloparsec scales, the arcs observed in some FRI jets have also been suggested to be due to changes in the injection rates from the source. ${ }^{54}$ 


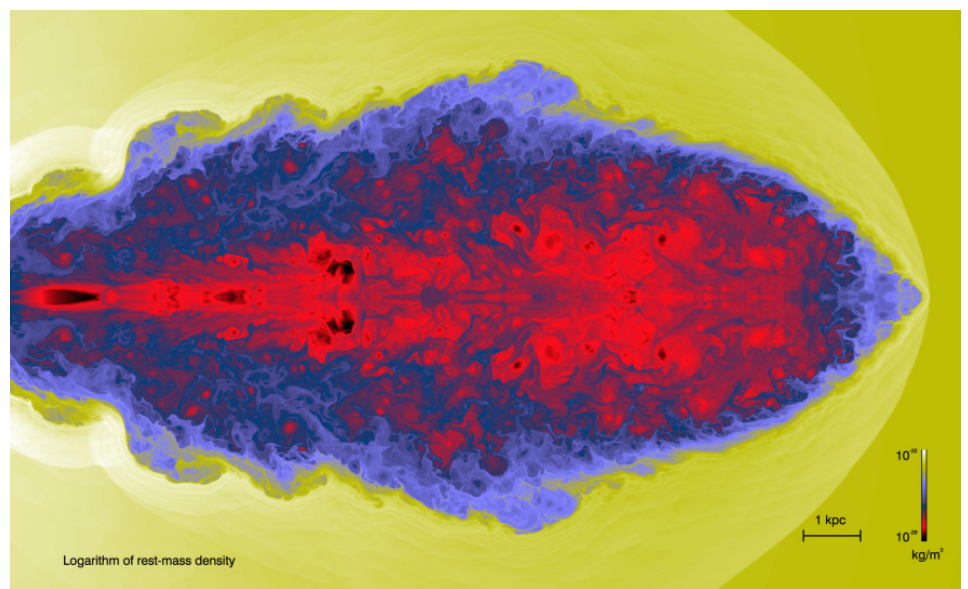

Fig. 5. Snapshot of rest-mass density from a long-term 2D simulation of a FRI-like jet evolving in a King-density profile. The jet is disrupted after non-linear pinching triggered by a strong reconfinement shock at $\simeq 1.5 \mathrm{kpc}$ from the injection in the numerical grid. The colour bar ranges from $10^{-32}$ to $10^{-25} \mathrm{~g} / \mathrm{cm}^{3}$. From ref. 42 .

Microquasar jets Inhomogeneities in the ambient medium can also have a strong effect on microquasar jets in high-mass X-ray binaries (HMXB) with typical powers $\left(10^{36-37} \mathrm{erg} / \mathrm{s}\right) .{ }^{55}$ Such inhomogeneities are encountered by the jet in the transition between the unshocked and shocked stellar wind from the massive companion. Another change of ambient medium occurs for a young HMXB still embedded in the SNR, at the transition between the shocked wind and the shocked SNR, and finally from the latter to the interstellar medium (ISM) through the shocked ISM. For the case of older HMXB, the system has moved away from the SNR or this has become very dilute and the region of interaction between the shocked stellar wind and the shocked ISM also implies a change in the conditions encountered by jets. In these density jumps jets are decelerated and need several thousand years to drill the shocked ISM before being able to propagate into the ISM. Proper motion of the HMXB provides a further destabilising mechanism, as the jet is impacted by the otherwise quasi-steady shocked stellar wind, propagating in the direction opposite to that of the proper motion.

Within the binary system, the stellar wind impacting the jet can also generate jet entrainment, deceleration and loss of collimation for jets with powers $\leq 10^{37} \mathrm{erg} / \mathrm{s}$, mainly if a strong reconfinement shocks that decelerate the flow occur in this region, as has been shown to be the possible. ${ }^{5,57}$ See also Figure 6.

\section{A global (and personal) view on the stability of relativistic jets}

In the previous sections, the main properties of instabilities that can affect the largescale morphology of relativistic jets, namely CDI and KHI, have been summarised. These have been proven to be disruptive enough to trigger efficient entrainment and 

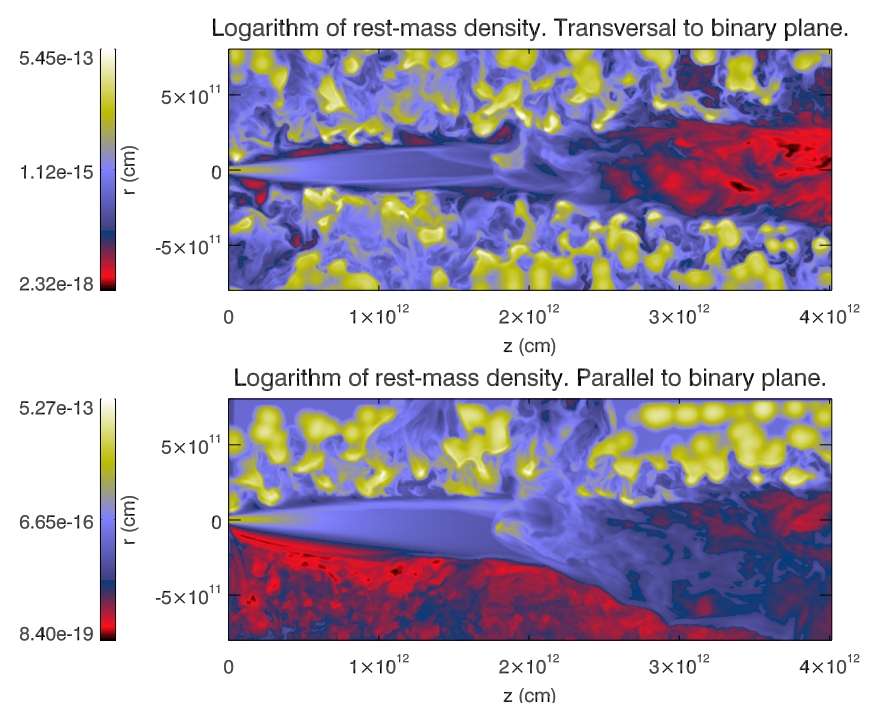

Fig. 6. Two perpendicular cuts along the axial direction of rest-mass density from a 3D simulation of a jet interacting with an inhomogeneous stellar wind in a HMXB. Units are cgs. From ref. 57.

decelerate jets. However, several mechanisms that may damp their growth and thus make relativistic jets stable have been given:

- CDI: Poloidal magnetic field, mildly relativistic sheaths and jet expansion.

- KHI: Thick shear-layers or surrounding winds, the decrease of the cocoon density with time and jet expansion.

Most importantly, it has been shown that relativistic jets are less sensitive to the growth of instabilities than classical jets, due to the saturation of their growth at the speed of light. In addition, resonant modes that can show up in fast jets surrounded by thin shear-layers, or simply the development of short wavelength modes as compared to the jet radius can result in little mixing, restricted to the boundaries of jets, and thus little loss of jet thrust and collimation. If one or several of these mechanisms play a role, jets can remain collimated along large distances, as it has been shown by simulations. Other mechanisms such as jet rotation or different configurations of the magnetic field are still to be explored in the relativistic regime.

The properties of jets and the media outside the leading bow-shock change with distance to the source. The cocoons surrounding jets show a homogeneous pressure in long-term simulations of jets due to their sound-speeds being larger than the expansion velocities. As the cocoon expands, its density and pressure drop and, as long as the properties of the jet do not change, it will become denser with respect to its environment, gaining extra stability. The only caveat is that when the cocoon is very under-pressured, strong reconfinement shocks may form. Nevertheless, even when jets are entrained and decollimated, they seem to preserve a large velocity 
that can be sub-relativistic, but could still be enough to keep the propagation of the jet to large scales.

In FRI jets, as compared to FRII's, it is more likely that non-linear processes like mass-loading from stars ${ }^{46}$ or strong reconfinement shocks ${ }^{42}$ are the cause of their disrupted morphologies. These jets have apparently similar velocities in the parsec-scales as the FRII's, ${ }^{59}$ so other intrinsic properties such as density, temperature or composition should play a role to explain their lower powers. They are observed to have an opening angle in the inner regions, but there is not any hint of growth of instabilities before the jet deceleration and loss of collimation. In hybrid sources in which the jet and counterjet appear to have different morphologies, ${ }^{48}$ it is possible that irregularities in the ambient medium are the cause of the difference, for jets with powers between those typical of FRIs and FRIIs. Only in one case an FRII classified jet with an apparently irregular structure in the largest scales, ${ }^{60}$ has been reported to develop instabilities that could cause the jet disruption. ${ }^{26}$ This implies that, under regular conditions, i.e., without strong non-linear perturbations, the stabilising mechanisms proposed by different authors in the last years and summarised here play a significant role. It has also been suggested that FRII jets can turn into FRI jets in the long term ${ }^{61}$ or after the end of an active phase, as numerical simulations show. ${ }^{62}$ However, this possibility should be matched to the relativistic velocities measured in FRI jets at the parsec scales.

The long-term stability of relativistic jets allows them to carry energy from very small to very large scales. The bow-shocks generated by extragalactic jets need $10^{7}-10^{8}$ years to reach distances of the order of hundreds of kpc. In the most powerful radio-sources, strong shocks are triggered in the ISM and ICM, which can change the evolution of the galaxies themselves by removal of ISM. This process can suppress star formation significantly and heating the surrounding gas, as shown by recent long-term simulations of relativistic jets. ${ }^{62}$ Thus, the importance of the study of this interaction and the evolution of the jet itself.

The research on jet stability in the next years should be addressed to investigate the effect of magnetic fields on the long-term evolution of jets. This should help to study, with input from observations and modelling, to which distance and extent the magnetic field is dynamically important and the role of instabilities in the eventual transfer of Poynting flux into kinetic energy of particles. In addition, more realistic situations should be explored, such as jet rotation and expansion. These works will certify or falsify the role of the stabilising mechanisms summarised in this contribution and could even add further ones, suggesting that there may be more than one answer to the problem of jet stability. Finally, it would be important to study, via detailed numerical simulations, the process of mixing and entrainment that jets undergo as a consequence not only of the growth of instabilities, but also of the interaction with stellar winds and clouds of cold gas. 


\section{Acknowledgements}

I acknowledge J.M. Martí, M. Hanasz and P.E. Hardee for sharing their understanding of jet physics with me during the last years. Financial support by the Spanish "Ministerio de Ciencia e Innovación" (MICINN) grants AYA2010-21322-C03-01, AYA2010-21097-C03-01 and CONSOLIDER2007-00050 is also acknowledged.

\section{References}

1. R. D. Blandford and R. L. Znajek, Mon. Not. R. Astron. Soc. 179, 433 (1977).

2. R. D. Blandford and D. G. Payne, Mon. Not. R. Astron. Soc. 199, 883 (1982).

3. B. D. Turland and P. E. G. Scheuer, Mon. Not. R. Astron. Soc. 176, 421 (1976).

4. R. D. Blandford and J. E. Pringle, Mon. Not. R. Astron. Soc. 176, 443 (1976).

5. A. Ferrari, E. Trussoni and L. Zaninetti, Astron. Astrophys. 64, 43 (1978).

6. P. E. Hardee, Astrophys. J. 234, 47 (1979).

7. P. E. Hardee, The stability of astrophysical jets, in Jets at all scales, Proc. of the 275 IAU Symposium, eds. G. E. Romero, R. A. Sunyaev and T. Belloni (Cambridge University Press, Cambridge, 2011), p. 41.

8. J. C. McKinney and R. D. Blandford, Mon. Not. R. Astron. Soc. 394, 126 (2009).

9. Y. Mizuno et al., Astrophys. J. 700, 684 (2009).

10. Y. Mizuno, P. E. Hardee and K. I. Nishikawa, Astrophys. J. 734, 19 (2011).

11. M. Sikora et al., Astrophis. J. 625, 72 (2005).

12. P. E. Hardee and J. A. Eilek, Astrophys. J. 735, 61 (2011).

13. S. S. Komissarov et al., Mon. Not. R. Astron. Soc. 394, 1182 (2009).

14. Y. Mizuno, P. Hardee and K. I. Nishikawa, Astrophys. J. 662, 835 (2007).

15. D. G. Payne and H. Cohn, Astrophys. J. 291, 655 (1985).

16. P. E. Hardee, Astrophys. J. 533, 176 (2000).

17. M. Birkinshaw, The stability of jets, in Beams and Jets in Astrophysics, ed. P. A. Hughes, (Cambridge University Press, Cambridge, 1991), p. 278.

18. M. Perucho, J. M. Martí and M. Hanasz, Astron. Astrophys. 443, 863 (2005).

19. P. E. Hardee, Astrophys. J. 318, 78 (1987).

20. A. P. Lobanov and J. A. Zensus, Science 294, 128 (2001).

21. P. E. Hardee, R. C. Walker and J. L. Gómez, Astrophys. J. 620, 646 (2005).

22. M. Perucho et al., Astron. Astrophys. 456, 493 (2006).

23. A. P. Lobanov et al., Publ. Astr. Soc. Jap. 58, 253 (2006).

24. M. Perucho and A. P. Lobanov, Astron. Astrophys. 469, L23 (2007).

25. M. Perucho, A. P. Lobanov and Y. Y. Kovalev, Physical Information Derived from the Internal Structure in Jets, in Approaching Micro-Arcsecond Resolution with VSOP-2, Astrophysics and Technologies ASP Conference Series, Vol. 402, eds. Y. Hagiwara, E. Fomalont, M. Tsuboi and Y. Murata (San Francisco, 2009), p. 349.

26. M. Perucho et al., in preparation.

27. M.-A. Aloy et al., Astrophys. J. 528, 85 (2000).

28. G. Bodo et al., Astron. Astrophys. 283, 655 (1994).

29. M. Hanasz, PhD. Thesis, (1995).

30. M. Hanasz, Kelvin-Helmholtz Instability of Relativistic Jets - the Transition from Linear to Nonlinear Regime, in Relativistic Jets in AGNs, eds. M. Ostrowski, M. Sikora, G. Madejski, M. Begelman (Krakow, 1997), p. 85.

31. M. Perucho et al., Astron. Astrophys. 427, 415 (2004).

32. M. Perucho, J. M. Martí and M. Hanasz, Astron. Astrophys. 427, 431 (2004).

33. M. Perucho et al., Phys. Rev. E 75, 6312 (2007). 
34. P. E. Hardee and P. A. Hughes, Astrophys. J. 583, 116 (2003).

35. V. Urpin, Astron. Astrophys. 385, 14 (2002).

36. P. E. Hardee, Astrophys. J. 664, 26 (2007).

37. M. Perucho et al., Astron. Astrophys. 519A, 41 (2010).

38. J. Xu, P. E. Hardee and J. Stone, Astrophys. J. 543, 161 (2000).

39. R. A. Daly and A. P. Marscher, Astrophys. J. 334, 539 (1988).

40. S. A. E. G. Falle, Mon. Not. R. Astron. Soc. 250, 581 (1991).

41. I. Agudo et al., Astrophys. J. Lett. 549, L183 (2001).

42. M. Perucho and J. M. Martí, Mon. Not. R. Astron. Soc. 382, 526 (2007).

43. A. T. Araudo, V. Bosch-Ramon and G. E. Romero, Astron. Astrophys. 522, 97 (2010).

44. M. V. Barkov, V. Bosch-Ramon and F. Aharonian, Astrophys. J. 724, 1517 (2010).

45. S. S. Komissarov, Mon. Not. R. Astron. Soc. 269, 394 (1994).

46. R. A. Laing and A. H. Bridle, Mon. Not. R. Astron. Soc. 336, 1161 (2002).

47. A. Hubbard and E. G. Blackman, Mon. Not. R. Astron. Soc. 371, 1717 (2006).

48. Gopal-Krishna and P. J. Wiita, Astron. Astrophys. 363, 507 (2000).

49. Z. Meliani, R. Keppens and B. Giacomazzo, Astron. Astrophys. 491, 321 (2008)

50. P. Rossi et al., Astron. Astrophys. 488, 795 (2008).

51. M. Kadler, E. Ros and M. Perucho, et al., Astrophys. J. 680, 867 (2008).

52. M. Perucho et al., Astron. Astrophys. 489, L29 (2008).

53. P. Mimica et al., Astrophys. J. 696, 1142 (2009).

54. R. A. Laing et al., Mon. Not. R. Astron. Soc. 386, 657 (2008).

55. V. Bosch-Ramon, M. Perucho and P. Bordas, Astron. Astrophys. 528, 89 (2011).

56. M. Perucho and V. Bosch-Ramon, Astron. Astrophys. 482, 917 (2008).

57. M. Perucho, V. Bosch-Ramon and D. Khangulyan, Astron. Astrophys. 512, L4 (2010).

58. M. Perucho and V. Bosch-Ramon, Astron. Astrophys., in preparation.

59. G. Giovannini et al., Astrophys. J. 552, 508 (2001).

60. C. A. Hummel et al., Astron. Astrophys. 266, 93 (1992).

61. Y. Wang et al., to appear in Mon. Not. R. Astron. Soc. [arXiv:1108.0558].

62. M. Perucho, V. Quilis and J. M. Martí, to appear in Astrophys. J. [arXiv:1107.5484]. 